
allemande

49-1 | 2017

Berlin 1957-1994

\title{
Ökonomische und soziokulturelle Ungleichheiten in Bezug auf einen ökologischen Wandel
}

Die Energiewende am Beispiel von Straßburg

Philippe Hamman

\section{(2) OpenEdition}

\section{Journals}

Édition électronique

URL : https://journals.openedition.org/allemagne/543

DOI : 10.4000 /allemagne. 543

ISSN : 2605-7913

\section{Éditeur}

Société d'études allemandes

Édition imprimée

Date de publication : 16 juin 2017

Pagination : 223-242

ISSN : 0035-0974

Référence électronique

Philippe Hamman, „Ökonomische und soziokulturelle Ungleichheiten in Bezug auf einen ökologischen Wandel", Revue d'Allemagne et des pays de langue allemande [Online], 49-1 | 2017, Online erschienen am: 16 Juni 2018, abgerufen am 02 Juni 2022. URL: http://journals.openedition.org/allemagne/543 ; DOI: https://doi.org/10.4000/allemagne.543 


\section{Ökonomische und soziokulturelle Ungleichheiten in Bezug auf einen ökologischen Wandel}

\section{Die Energiewende am Beispiel von Straßburg}

- Philippe Hamman*

\section{Einleitung}

Bei den aktuellen Herausforderungen im Umweltschutz und in der nachhaltigen Entwicklung wird in unserer manchmal als "postpolitisch “ bezeichneten Zeit ${ }^{(1)}$ oft von einer „Konsenspolitik“ ausgegangen: Wer könnte ernsthaft gegen die Auseinandersetzung mit der Zukunft unseres Planeten sein, der von Klimaerwärmung, Naturkatastrophen und anderen „globalen“ Risiken bedroht wird? Diese Einstellung übersieht jedoch die zahlreichen Kontroversen hinsichtlich dieser Zukunft ${ }^{(2)}$. Geht es darum, sich in unserer immer stärker mit ihrer Endlichkeit konfrontierten Welt, deren Ressourcen nicht unerschöpflich sind und teilweise vom Menschen bereits unwiederbringlich verbraucht wurden, von der herrschenden Ansicht zu befreien, das ewige Wirtschaftswachstum sei auch ein ewiger Fortschrittsmotor? ${ }^{(3)}$ Oder sind Forschung und Technik in der Lage, "nachhaltige“, umweltkompatible Konzepte zu erfinden, insbesondere unter dem Schlagwort des „grünen Wachstums“? ${ }^{(4)}$

* Professor für Stadt- und Umweltsoziologie am Institut für Stadtsoziologie und regionale Raumplanung der Fakultät für Sozialwissenschaften, Universität Straßburg: <phamman@unistra.fr>.

1 Slavoj Zızek, The Ticklish Subject: The Absent Centre of Political Ontology, London, Verso, 1999; Erik Swyngedouw, „The Antinomies of the Postpolitical Cities: In Search of Democratic Politics of Environmental Production“, International Journal of Urban and Regional Research, 33/3 (2009), S. 601-620.

2 Siehe die kritische Medienanalyse von Jean-Baptiste Сомву, La question climatique. Genèse et dépolitisation d'un problème public, Paris, Raisons d'agir, 2015.

3 Nicholas Georgescu-Roegen, The Entropy Law and the Economic Process, Cambridge, Harvard University Press, 1971; Federico Demaria, François Schneider, Filka Sekulova, Joan Martinez-Alier, „What is Degrowth? From an Activist Slogan to a Social Movement“, Environmental Values, 22 (2013), S. 191-215.

4 Arthur P. J. Mol, David A. Sonnenfeld, Gert Spaargaren (Hg.), The Ecological Modernisation Reader: Environmental Reform in Theory and Practice, New York, Routledge, 2009; Ian BAILEY, 
Während des Weltgipfels zu Umwelt und Entwicklung in Rio im Jahr 1992, wurde die nachhaltige Entwicklung grundsätzlich als Bruch mit den Prinzipien des Wirtschaftswachstums entworfen, wobei gleichzeitig genügend Spielraum gelassen wurde, um den verschiedenen Akteuren und ihren unterschiedlichen Zielsetzungen ihre jeweils eigene Aneignung zu ermöglichen ${ }^{(5)}$. Das nachhaltige Wachstum wurde infolgedessen in den Jahren 1990-2000 ein erfolgreiches Handlungsrepertoire, dessen praktische Umsetzbarkeit sich bewährt hat, indem es den Bezugsrahmen der Gesellschaftspolitik in Europa verändert hat ${ }^{(6)}$. Doch die Wirtschaftskrise von 2007-2008 hat die Schwachstellen dieses Konzeptes der nachhaltigen Entwicklung offengelegt, das schematisch eine technische und wirtschaftliche Interpretation der Nachhaltigkeit einer ökologischen und sozialen entgegensetzt ${ }^{(7)}$. Wäre es folglich nicht sinnvoller, die Möglichkeit eines Rückzugs in Betracht zu ziehen, im Sinne einer „Entkolonialisierung der natürlichen Systeme" durch einen Rückzug der Technik, wie sie von Marina Fischer-Kowalski et al. erwogen wurde ${ }^{(8)}$, zusammen mit einer Förderung des sozialen Zusammenhalts?

Die Problematik der Systeme zur Nutzung der erneuerbaren Energien verdeutlicht diese Fragestellungen angesichts der in der "Roadmap 2050“ beschriebenen Ziele der EU, 80\% Einsparung bei den $\mathrm{CO}_{2}$-Emissionen, bezogen auf den Wert von 1990, zu erreichen ${ }^{(9)}$. Auch wird deutlich, dass die nachhaltige Entwicklung nicht bloß ein "großes Thema" auf globaler Ebene ist; im Gegenteil, sie konkretisiert sich gerade in den Interaktionen zwischen globaler und lokaler Ebene. Verbreitung und lokale Umsetzung von Fachwissen und lokalem Wissen spielen hier eine essentielle Rolle ${ }^{(10)}$, sowohl auf Ebene der individuellen, als auch der kollektiven Lebensumstände. Dies unterstreicht

Federico Caprotti, „The Green Economy: Functional Domains and Theoretical Directions of Enquiry“, Environment and Planning A, 46/8 (2014), S. 1797-1813.

5 Phil McManus, „Politics, Stories and Discourses of Sustainability“, Environmental Politics, 5/1 (1996), S. 48-73; Lamont C. Hempel, „Conceptual and Analytical Challenges in Building Sustainable Communities“, in: Daniel A. Mazmanian, Michael E. Kraft (Hg.), Towards Sustainable Communities, Cambridge, MIT Press, 1999, S. 43-74; Edwin ZACCAI, „Over Two Decades in Pursuit of Sustainable Development: Influence, Transformation, Limits“, Environmental Development, 1/1 (2012), S. 79-90.

6 Es kann sich um ein umrissenes Set an Aktivitäten zur Erreichung eines nachhaltigen Wachstums handeln, und/oder um die Vermeidung bestimmter Aktivitäten, die einem Mehr an Nachhaltigkeit im Wege stehen: Philippe Hamman, „Urban Sustainable Development and the Challenge of French Metropolitan Strategies“, Urban Research and Practice, 2/2 (2009), S. 138-157; Philippe Hamman, Sociologie urbaine et développement durable, Bruxelles, De Boeck, 2012.

7 Hamil Pearsall, Joseph Pierce, Robert Krueger, „Whither Rio+20? Demanding a Politics and Practice of Socially Just Sustainability“, Local Environment, 17/9 (2012), S. 935-941; Philippe HAMman, Virginie Anquetin, Céline Monicolle, „Contemporary Meanings of the ,Sustainable City‘. A Comparative Review of the French and English-Language Literature“, Sustainable Development, 24 (2016), DOI: 10.1002/sd.1660.

8 Marina Fischer-Kowalski, Helmut Haberl, Walter Hüttler, Harald Payer, Heinz Schande, Verena Winiwarter, Helga ZangerL-Weisz, Gesellschaftlicher Stoffwechsel und Kolonisierung von Natur, Amsterdam, G+B Verlag Fakultas, 1997.

9 https://ec.europa.eu/energy/sites/ener/files/documents/2012_energy_roadmap_2050_en_0.pdf (letzter Zugriff am 19.11.2016).

10 Susan Baker, Katarina Eckerberg (Hg.), In Pursuit of Sustainable Development: New Governance Practices at the Sub-National Level in Europe, London, Routledge, 2008; Ph. Hamman, Sociologie urbaine et développement durable (Anm. 6). 
den Platz des Sozialen in dem, was zuweilen zu Unrecht primär als ein technisches Thema wahrgenommen wird, besonders, was die Energieerzeugung angeht ${ }^{(11)}$.

Die Klimaerwärmung ist heute in der Politik angekommen und ist zu einem zentralen Thema nachhaltiger Gesellschaftspolitik geworden ${ }^{(12)}$, was zugleich das Risiko einer ausschließlichen Sicht aus der Perspektive der politischen Führung birgt ${ }^{(13)}$. Die Instrumente der öffentlichen Politik sind dabei Träger einer bestimmten Sicht der sozialen Welt und bestimmter Interpretationsschemata ${ }^{(14)}$ : Sie führen Konzepte der Energiewende ein, welche die Beziehungen zwischen Ökologie und Ökonomie unterschiedlich definieren. 2012 wurde beim Weltgipfel Rio+20 die progressive Ablösung der fossilen Energien beschlossen. Zugleich wurde die Energiewende zu einem Motor der Green Economy gemacht ${ }^{(15)}$. Die erneuerbaren Energien gehorchen demzufolge den Gesetzen des Marktes, da sie einen neuen Wirtschaftssektor entwickeln. Beispielhaft kann hier das französische Gesetz zur „Energiewende für ein grünes Wachstum“ genannt werden, das im November 2014 mit dem Ziel verabschiedet wurde, die Entwicklung technologischer Innovationen zur Förderung weniger energieintensiver Lebensweisen zu vereinfachen, ohne die Wirtschaft nachteilig zu beeinflussen.

Es können drei zentrale Logiken des Strommarktes identifiziert werden: eine korporatistische Versorgungslogik, eine wettbewerbsorientierte Marktlogik und eine partizipative Umweltlogik ${ }^{(16)}$. Vom soziologischen Standpunkt aus können in diesem Kontext der handelnden Akteure drei Analyse-Ebenen miteinander in Verbindung gesetzt werden: die Branchen, welche die erneuerbaren Energien verbreiten; der Inhalt dessen, was verbreitet wird; sowie die Praktiken und Aneignungsgrade, welche die Einführungsmethoden erlauben ${ }^{(17)}$. Die Frage der Aneignung beleuchtet auch die der

11 Guillaume Christen, Philippe Hamman, Matthias Jehling, Maurice Wintz (Hg.), Erneuerbare Energien-Systeme in Deutschland und Frankreich - Systèmes énergétiques renouvelables en France et en Allemagne, Paris, Orizons, 2014, S. 279-318; Guillaume Christen, Philippe Hamman, „Associer les habitants à la transition écologique: Quelle dimension participative des projets d'énergies renouvelables en Alsace?", Cahiers de recherche sociologique, 58 (2015-2016), S. 119-137, DOI: 10.7202/1036209ar; Philippe Hamman, „Durabilité et lien social: transition et transaction dans l'expérimentation“, SociologieS, juin 2016, https://sociologies.revues.org/5384. Siehe auch Marie-Christine ZÉLEM, Christophe Beslay (Hg.), Sociologie de l'énergie. Gouvernance et pratiques sociales, Paris, CNRS Éditions, 2015.

12 Cyria Emelianoff, „Local Energy Transition and Multilevel Climate Governance: The Contrasted Experiences of Two Pioneer Cities (Hanover, Germany, and Växjö, Sweden)“, Urban Studies, 51/7 (2014), S. 1378-1393; Stefanie RössLer, „Klimawandelgerechte Stadtentwicklung durch grüne Infrastruktur“, Raumforschung und Raumordnung, 73/2 (2015), S. 123-132.

13 Vincent BÉAL, Gilles Pinson, „From the Governance of Sustainability to the Management of Climate Change: Reshaping Urban Policies and Central-Local Relations in France“, Journal of Environmental Policy and Planning, 17/3 (2015), S. 402-419.

14 Pierre Lascoumes, Patrick Le Galès, „From the Nature of Instruments to the Sociology of Public Policy Instrumentation“, Governance, 20/1 (2007), S. 1-21.

15 Pearsall/Pierce/Krueger, „Whither Rio+20?“ (Anm. 7); Christian Schulz, Ian Bailey, „The Green Economy and Post-Growth Regimes: Opportunities and Challenges for Economic Geography“, Geografiska Annaler: Series B, Human Geography, 96/3 (2014), S. 277-291.

16 Thomas Kern, „Die Umweltbewegung und der Wandel der institutionellen Logik auf dem Strommarkt“, Zeitschrift für Soziologie, 43/5 (2014), S. 322-340.

17 Energieerzeugung und -verteilung beruhen auf einer Vielfalt von interagierenden Akteuren: Entscheider, Kollektivitäten, Verbraucher usw. Die Nutzung und die Verbreitung einer Energiequelle gleicht 
Partizipation, die als Pfeiler der nachhaltigen Entwicklung und ihrer lokalen Umsetzung identifiziert wurde ${ }^{(18)}$ : Es geht dabei um die Sensibilisierung und Einbindung der Einwohner, insbesondere, was den Einsatz von Technologien betrifft, die zur ökologischen Energiegewinnung beitragen sollen. Dieser Artikel befasst sich im Detail mit der räumlichen Umsetzung neuer Formen der Ungleichheit und stellt dabei die Frage: Überwindet, verfestigt oder vertieft die Einführung der erneuerbaren Energien die sozialen Gräben in der Stadt?

In diesem Sinne untersuchen wir die umweltbezogenen sozialen Ungleichheiten anhand der Fähigkeiten der Akteure, sich bei Umweltprojekten vor Ort zu engagieren und zu integrieren: Ist das etwas grundlegend „Neues“ oder eine weitere Spielart bereits existierender sozialer Ungleichheiten? Dazu greifen wir auf qualitative und quantitative Vor-Ort-Umfragen im urbanen Umfeld zurück, die von 2012 bis $2014 \mathrm{im}$ Rahmen eines europäischen Forschungsprojektes in der Metropolregion Straßburgs durchgeführt wurden. Wir haben uns auf zwei Standorten fokussiert (siehe unten), und eine quantitative Umfrage durchgeführt (Sampling basiert auf der Zusammensetzung der Berufsgruppen in beiden Fällen; wir erhalten 300 verwertbare Antworten, davon 150 pro Standort - Die Antworten wurden unter Verwendung von SAS und CALMAR-Software ${ }^{(19)}$ analysiert), verbunden mit einem Dutzend Leitfadeninterviews mit den betroffenen Einwohnern (von den Antworten auf den quantitativen Fragebögen ausgewählt).

Ziel ist eine neue Herangehensweise an das Thema der umweltbezogenen Ungleichheiten, welche sich nicht auf die Ungleichheiten bei der Belastung durch Umweltschäden fokussiert, sondern die konkreten Zusammenhänge zwischen umweltbezogenen Ungleichheiten und dem Diskurs der Partizipation untersucht, hinter dem sich „ÖkoInnovationen“ verbergen können, die in Wirklichkeit Präskriptionen gleichkommen (1. Teil). Wir zeigen somit auf, dass die Entstehung umweltbezogener Ungleichheiten, welche die Auswirkungen des sozialen Gefälles in der Stadt verschärfen, in hohem Maße auf den unterschiedlichen Bezug der Einwohner zu den technischen Instrumenten, welche die Realisierung einer Energieproduktion aus erneuerbaren Quellen möglich machen sollen, zurückzuführen ist (2. Teil). Dies ermöglicht, die Nachhaltigkeit aus

einem soziotechnischen System, das auf drei interagierenden Säulen gründet: Die Energiequelle, die technischen Mittel, dank derer die Ressource nutzbar gemacht wird (beispielsweise Sonnenenergie) und das technisch-wirtschaftliche Modell: Guillaume Christen, Philippe Hamman, Transition énergétique et inégalités environnementales: énergies renouvelables et implications citoyennes en Alsace, Strasbourg, Presses universitaires de Strasbourg, 2015. Siehe auch Paul Burger et al., „Advances in Understanding Energy Consumption Behavior and the Governance of its Change - Outline of an Integrated Framework“, Frontiers in Energy Research, 3 (2015), DOI: 10.3389/fenrg.2015.00029.

18 Meg Holden, „Public Participation and Local Sustainability: Questioning a Common Agenda in Urban Governance“, International Journal of Urban and Regional Research, 35/2 (2011), S. 312-329; Christopher V. Hawkins, XiaoHu WAng, „Sustainable Development Governance. Citizen Participation and Support Networks in Local Sustainability Initiatives“, Public Works, Management and Policy, 17/1 (2012), S. 7-29; Ph. Hamman, Sociologie urbaine et développement durable (Anm. 6).

19 „Statistical Analysis System“ und „calages à la marge“ (gewichtete Stichprobe): http://www.insee.fr/fr/ methodes/default.asp?page=outils/calmar/accueil_calmar.htm (letzter Zugriff am 19.11.2016). Diese Studie wäre ohne die Mitwirkung von Guillaume Christen, Camille Cretté und Céline Monicolle (Forschungszentrum SAGE, UMR 7363), die wir an dieser Stelle danken möchten, nicht denkbar gewesen. 
einer grassroot sozialen Perspektive zu betrachten, und in der Technologie nicht unbedingt „die“ Antwort auf die aktuelle Umweltkrise zu sehen (3. Teil). Damit unterscheidet sich dieser Artikel von einem großen Teil soziologischer Studien zur Energiewende, welche sich vor allem mit den Möglichkeiten der Umsetzung, selten aber mit der kritischen Reflektion des Konzepts der Partizipation/Bürgerbeteiligung beschäftigen ${ }^{(20)}$.

\section{Öko-Innovationen zwischen Anreiz und Präskription}

Die ersten sozialwissenschaftlichen Forschungsarbeiten, welche die Beziehung zwischen sozialen Ungleichheiten und Umwelt problematisieren, stammen aus den Vereinigten Staaten. Sie befassen sich vor allem mit der „Umweltgerechtigkeit“, bei der die Faktoren Umwelt und Natur als Grundsteine für eine bessere soziale Gerechtigkeit verstanden werden. Haupthypothese ist, dass die sozialräumliche Ausgrenzung ethnischer Minderheiten, die in eigenen Stadtvierteln angesiedelt werden, mit einer weiteren, umweltbezogenen Ausgrenzungsform einhergeht. Die Minderheiten sind Umweltbelastungen stärker „,ausgesetzt“, weil sie in der Nähe von großen Verkehrsachsen, Schienen oder Industrieanlagen leben. Besonders Robert D. Bullard hat am Beispiel Bodenverschmutzung die verstärkte Belastung von Bevölkerungsgruppen aufgezeigt, die aus benachteiligten sozialen Schichten stammen und/oder ethnischen Minderheiten angehören (besonders Afroamerikaner) ${ }^{(21)}$.

In der Folge haben manche Forscher zwischen umweltbezogenen Ungleichheiten und ökologischen Ungleichheiten inhaltlich unterschieden ${ }^{(22)}$. Bei ökologischen Ungleichheiten wird das Soziale in Verbindung zur Umwelt gesetzt und es werden die sozialen Ungleichheiten gegenüber der Umwelt thematisiert. Dabei geht es um Unterschiede bei Wissen und Verhaltensweisen der verschiedenen Akteure in Bezug auf die Umwelt sowie um den unterschiedlichen Zugang zu soziokulturellen Ressourcen, die notwendig sind, um an Umwelttechnologien teilhaben zu können ${ }^{(23)}$. Die Beziehungen

20 Diese Forschungsarbeit wurde mit Unterstützung des Europäischen Fonds für regionale Entwicklung (EFRE) und dem Interreg IV Programm für die Region Oberrhein in Zusammenarbeit mit der Wissenschaftsoffensive der Trinationalen Metropolregion Oberrhein (TMO): Projekt C31 „Plan-Énergies renouvelables“ („Plan-EE“, Entwicklung eines Planungstools zur Einführung der erneuerbaren Energien in der Region Oberrhein, 2012-2015) durchgeführt. Weiterführende Informationen finden Sie auf der Website: http://www.plan-ee.eu/.

21 Robert D. Bullard, Dumping in Dixie: Race, Class, and Environmental Quality (1990), Boulder, CO, Westview Press, 2000.

22 Mathieu Durand, Sylvy JAGLin, „Inégalités environnementales et écologiques: quelles applications dans les territoires et les services urbains?“, Flux, 89-90 (2012), S. 4-14. Siehe auch Aurélie Choné, Isabelle Најек, Philippe Намmаn (Hg.), Guide des Humanités environnementales, Villeneuve d'Ascq, Presses universitaires du Septentrion, 2016, Teil 2.

23 Es ist möglich, die technischen Entscheidungen auf soziale Determinismen wie dem Werdegang der Akteure, ihre Ausbildung, ihre sozialen Beziehungen und ihre Verankerung in verschiedenen Strukturen zurückzuführen. Gleichzeitig bildet das Dispositiv einen Rahmen, der es den Akteuren ermöglicht, sich das Instrument ihrer Praxis entsprechend anzueignen. Die Herausforderung ist, zu erfahren, ob die Akteure die Möglichkeit haben, diesen Rahmen zu verlassen und das Wissen und die Handlungsrepertoires stärker mitzubestimmen. Siehe Philippe Hamman, „Die Aneignungsungleichheiten in Hinsicht auf die regionale Energieerzeugung im Zeitalter der ökologischen Wende. Soziologische Analyse eines kooperativen Instrumentes im Bereich ,Bürgerwindkraft‘ im Elsass“, Revue d’Allemagne et des Pays de langue allemande, 48/1 (2016), S. 143-164; Guillaume Christen, Philippe 
zwischen Partizipation und Umwelt spielen eine entscheidende Rolle, da der offizielle institutionelle Diskurs die Bürger dank verschiedener Anreize an der Nachhaltigkeit beteiligen will ${ }^{(24)}$. Doch einfach zu behaupten, die Einwohner in die Energiewende einzubeziehen, reicht nicht: Die praktischen Modalitäten, welche die Partizipation regeln, können deren Handlungsmöglichkeiten einschränken. Die Bürger können nicht alle gleichermaßen zum Gemeingut einer „grünen Energieproduktion“ beitragen ${ }^{(25)}$. Insbesondere, der zentralgesteckte bzw. vorformatierte Aktionsrahmen schließt die Bevölkerung oft von der Möglichkeit aus, ihr eigenes Gebrauchswissen zum Thema Energie einzubringen ${ }^{(26)}$.

Heute beinhalten die Technologien zur Energieerzeugung aus erneuerbaren Quellen, welche die industriellen Energieerzeuger den Privatkunden und Pächtern anbieten, partizipative Instrumente zur Sensibilisierung der Akteure. Diese Anreize sollen zuvor verborgene Fragen sichtbar machen, deren Aneignung sich ursprünglich dem Bürger entzieht. Wie von Ulrich Beck gezeigt, sind die postmodernen Risiken, wie zum Beispiel der Klimawandel, nicht ohne weiteres sichtbar ${ }^{(27)}$. Ihre Konkretisierung wird erst durch eine technische und wissenschaftliche Beschreibung ermöglicht. Es geht folglich darum, die Energiewende greifbar zu machen, indem sie im alltäglichen Handeln verankert wird ${ }^{(28)}$.

Um diese Dimension zu untersuchen, befassen wir uns mit zwei signifikanten Fällen des Straßburger Stadtgebiets. Beim Ersten geht es um ein Photovoltaikprojekt in Plobsheim, einem Straßburger Vorort mit 4.110 Einwohnern im Jahr 2012, das sich an private Hausbesitzer richtet. Der Zweite betrifft die Erneuerung eines Wärmenetzes in einem Sozialbauviertel im Norden von Straßburg, der Cité de l'Ill. Es wurde in den 60er Jahren gebaut und zählt heute an die 6.500 Einwohner; die Technologie will sie mit Hilfe von sogenannten „Öko-Reflexen“-Anreizen (Heizungsthermostat einstellen usw.) für die neue Heizart gewinnen und ihr Verhalten im Alltag verändern. Tatsache ist, die Verfügungsrechte von Eigentümern und Mietern (und damit auch die Planungs- und Mitbestimmungshorizonte) unterscheiden sich maßgeblich voneinander. Dazu werden wir die ungleichen Partizipationschancen auch mit unterschiedlichen Wissensbeständen erklären.

Hamman, „Quelle participation habitante dans les projets locaux d'énergie renouvelable? Retour sur un dispositif d'énergie ,citoyenne dans une commune alsacienne“, in: Pascal Tozzi (Hg.), Villes et quartiers durables: la place des habitants, Bordeaux, Carrières sociales Éditions, 2016, S. 461-477.

24 M. Holden, „Public Participation and Local Sustainability“ (Anm. 18); Ph. Hamman, Sociologie urbaine et développement durable (Anm. 6), S. 109-131.

25 Gavin Brown, Peter Kraftl, Jenny Pickerill, Caroline Upton, „Holding the Future Together: Towards a Theorisation of the Spaces and Times of Transition“, Environment and Planning A, 44/7 (2012), S. 1607-1623.

26 Ph. Hamman, „Die Aneignungsungleichheiten auf die regionale Energieerzeugung“ (Anm. 23); Christen/Hamman, „Quelle participation habitante“ (Anm. 23); Guillaume Faburel, „Inégalités environnementales“, in: Choné/Hajek/Hamman, Guide des Humanités environnementales (Anm. 22), S. 529-543.

27 Ulrich BECK, Risikogesellschaft. Aufdem Weg in eine andere Moderne, Frankfurt am Main, Suhrkamp, 1986.

28 Siehe auch in diesem Sinne, was Kernenergie anbelangt, Caroline Kramer, Simon Kretz, „Wie bewerten junge Menschen an Standorten von Kernkraftwerken die Kernenergie? Eine empirische Studie“, atw, International Journal of Nuclear Power, 59/12 (2014), S. 697-700. 


\section{Die Anreize für Privateigentümer}

In Frankreich können die privaten Hausbesitzer sich zwischen zwei Formen der Solartechnik entscheiden: Photovoltaik und Photothermik. Die Photovoltaikanlagen sind mit dem zentralen Stromnetz verbunden. Es gibt eine Abnahmegarantie und einen garantierten Mindestpreis für den erzeugten Strom, den Électricité de France (EDF) dem Hausbesitzer zahlen muss. Die Photothermikanlagen arbeiten hingegen in einem geschlossenen Kreis (Warmwasser für die Sanitäranlagen). Generell werden diese Anlagen durch verschiedene Maßnahmen gefördert. Die Gemeinde oder die Region kann sogenannte Energieschecks vergeben, insbesondere im Rahmen des Programms Je rénove $B B C$ („Ich mache mein Haus zum Niedrigenergiehaus“). Nationale Hilfen gibt es beim Dispositiv J'éco-rénove, j’économise („Ich saniere ökologisch und spare“), das 2013 gestartet wurde, um Sanierungen zur Energieeinsparung zu fördern ${ }^{(29)} .2016$ beinhalten diese Maßnahmen einen zinsfreien Kredit, eine Prämie für den Hausbesitzer und eine Steuergutschrift für die Energiewende sofern Wärmedämmungsmaßnahmen durchgeführt werden und Heizungen verbaut werden, die erneuerbare Energien nutzen.

Viele Einwohner von Plobsheim berichten, häufig von Photovoltaikanbietern kontaktiert zu werden: „Ja, oft gehen Vertreter von Tür zu Tür oder werfen einen Flyer in den Briefkasten. Wir werden auch regelmäßig angerufen und gefragt, ob wir Interesse hätten“ (aus dem Interview mit einem Einwohner von Plobsheim, April 2014). In der Gemeinde haben 2014 von den 120 von uns befragten Hausbesitzern 18 eine Anlage zur Nutzung regenerativer Energien installiert, hauptsächlich Holzenergieanlagen oder Photovoltaikanlagen. Die geäußerten Beweggründe sind vor allem wirtschaftlicher Natur: es geht um die Steuergutschrift und die Senkung der Stromkosten.

\section{Die Anreizpolitik im Sozialwohnungsviertel: Eine auf Verbrauchssenkung beschränkte Partizipation}

Die Umstellung von Heizsystemen bietet die Gelegenheit, die Material- und Energieströme, die in die Stadt und wieder hinaus fließen, zu relokalisieren ${ }^{(30)}$. Dieser Lokalisierungsprozess kann als Wille verstanden werden, den Kreislauf zwischen Produktion und Verbrauch in einem kleinen Versorgungsnetz zu schließen. Die Fernwärme bietet einen guten Hebel zur Einführung erneuerbarer Energien, vor allem wenn man bedenkt, dass 2012 der Energiebedarf aller Gebäude auf 68,7 TOE (Tonnen Öl-Äquivalent) geschätzt wurde, das heißt auf 44,5\% des französischen Endenergieverbrauchs ${ }^{(31)}$. Die Fernwärmenetze entsprechen vollumfänglich dem Konzept der „nachhaltigen Stadt“ in dem der Einwohner als Akteur seines eigenen Lebensrahmens anerkannt wird, vor allem bei großen Wohnanlagen. In Wirklichkeit werden die Einwohner mithilfe eines umfassenden Regelwerks bei der Reduzierung ihres Energieverbrauchs „unterstützt“, das sie zur Annahme sog. Öko-Verhaltensweisen bewegen soll.

29 http://renovation-info-service.gouv.fr/ (letzter Zugriff am 19.11.2016).

30 Sabine Barles, L'invention des déchets urbains. France: 1790-1970, Seyssel, Champ Vallon, 2005; Christopher A. Kennedy et al., „Energy and Material Flows of Megacities“, Proceedings of the National Academy of Sciences, 112/19 (2015), S. 5985-5990, DOI: 10.1073/pnas.1504315112.

31 Französisches Ministerium für Ökologie, Nachhaltigkeit und Energie, Energiebilanz Frankreichs 2012, Juli 2013. 
In der Cité de l'Ill wird das ursprüngliche Fernwärmenetz durch ein Kraft-WärmeKopplungs-Holzheizkraftwerk ergänzt, und die Umstellung auf das neue Heizsystem wird von Gebäudesanierungsmaßnahmen zur Steigerung der Wärmeeffizienz begleitet, die sich über drei Jahre erstrecken (2012-2015); sie beinhalten das Dämmen der Dachböden $\left(19.500 \mathrm{~m}^{2}\right)$, die Fassadendämmung $\left(104.100 \mathrm{~m}^{2}\right)$ und das Verlegen von Thermostatventilen (11.278 Stück). Ziel dieser Maßnahmen ist eine Energieeinsparung von $42 \%{ }^{(32)}$. Diese Sanierungsmaßnahmen sind für den Sozialwohnungsträger, der einem Dienstleister seine „grüne“ Energie abkaufen wird, von großer Bedeutung. Das Wärmenetz wurde im Jahr 2000 an einen Energieversorger, Idex, übertragen, der auch mit dem Betrieb des Wärme-Kopplungs-Holzheizkraftwerks beauftragt wurde. Er ist für die Versorgung des Kraftwerks zuständig, die Wärmeerzeugung und die Wärmeversorgung sowie für den Unterhalt des Netzes. Der Sozialwohnungsträger hat folglich ein Interesse daran, den Energieverbrauch an der Quelle zu reduzieren.

Die Energiepolitik ermutigt die Bürger, für das globale Anliegen des Umweltschutzes und gegen die Energieverschwendung einzutreten: dafür dringen die Instrumente bis in die Privatsphäre vor, in den Kreis der Familie und in die Verbrauchsgewohnheiten der Menschen. Unter dem Motto „Renov’Ill: Gut leben in der eigenen Wohnung“ („Rénov'Ill: bien vivre dans son logement“) führt der Sozialwohnungsträger in der Cité de l'Ill eine Kampagne mit dem Namen „Öko-Einstellung“ („Éco-attitude“) durch, die eine Reihe von Vorschriften beinhaltet. Diese betreffen zum Beispiel die mechanisch gesteuerte Belüftung, welche die manuelle Belüftung der Räume ablöst: Hier findet ein kompletter Resozialisierungsprozess statt, der nicht selbstverständlich ist, und der die früheren Vorschriften zur „gesunden“ Lüftung durch Öffnen der Fenster ersetzt ${ }^{(33)}$.

Diese Technologien haben den Anspruch, den Bewohnern der Sozialwohnungen dazu zu verhelfen, ihren Energieverbrauch durch die Aneignung neuer Normen und Verhaltensweisen zu reduzieren. Deutlich wird hier ein weitergefasstes Paradoxon der urbanen Nachhaltigkeitspolitik: Während einerseits zu einer aktiven Teilnahme der Bürger bei den Veränderungen des Alltags aufgerufen wird (Mülltrennung, Energieverbrauch usw.), wird andererseits das Bild des passiven Verbrauchers heraufbeschworen, der erst mit pädagogischen Methoden „erzogen“ werden muss - wie ein Kind in der Schule. Obwohl die neuen Verhaltensweisen, die es anzunehmen gilt, nicht als Verpflichtungen oder Zwänge dargestellt werden, sind sie dennoch als „Erziehungsmaßnahme“ gedacht. Die verschiedenen Aktivitäten (Dusche, Heizung, Geschirrspülen, Beleuchtung) werden alle im Lichte des Diskurses der individuellen Verantwortung für die bewußte Energienutzung betrachtet.

Die in der Cité de l'Ill verteilten Infobroschüren fokussieren sich vor allem auf die Heizung, die den größten Teil des Energieverbrauchs ausmacht. Die Einwohner erhalten eine Anleitung, um ihnen die „rationale und rationelle“ Benutzung des Thermostats nahezubringen, mit einer vorgeschriebenen Maximaltemperatur von $20^{\circ} \mathrm{C}$, die als „Komforttemperatur“ beschrieben wird. Die Anleitung fungiert auch als Nachschlagewerk in Sachen „Best Practices“ beim Verbrauch von Warmwasser im Bad, das

32 http://www.habitationmoderne.org/fr/Habitation-Moderne-3.html (letzter Zugriff am 19.11.2016).

33 Philippe Hamman, Cécile Frank, Marie Mangold, „Les trajectoires de conversion écologique face aux enjeux socio-économiques du ,logement durable‘ en France“, VertigO, 14/2 (2014), http://vertigo. revues.org/15018 (letzter Zugriff am 19.11.2016). 
auch mit dem Kraft-Wärme-Kopplungs-Holzheizkraftwerk erzeugt wird. Dabei wird empfohlen, anstatt zu baden, lieber zu duschen, und zwar am besten innerhalb der „optimalen Dauer“ von 4 Minuten ${ }^{(34)}$.

Bei den Broschüren wird der Begriff der „Partizipation“ von der Behörde verwendet, um Praktiken der Energieersparnis durchzusetzen. Die Beziehung zwischen Eigentümer und Mieter ist asymmetrisch angelegt und wurde so gestaltet, dass der Sender die Verhaltensweisen der Empfänger lenkt. So sind die Mieter nicht mehr in der Lage, nach eigenem Gutdünken das Thermostat zu verstellen, da die Maximaltemperatur direkt von der Zentrale aus festgelegt wird. Die Umstellung stützt sich folglich auf eine vertikal organisierte Top-down-Steuerung, die einen normierten Gebrauch der Ressource vorschreibt. Im Kontext eines Sozialwohnviertels können solche Instrumente zu neuen Stigmatisierungsskriterien gegenüber den sozial Schwachen führen sowie zu einer Bevormundung der schwächsten Haushalte, denen ein verschwenderischer Lebensstil und das Versagen bei der Steuerung ihres Energieverbrauchs unterstellt wird, bei gleichzeitiger Hilfe, ihnen in ihrem eigenen materiellen Interesse zu einem besseren Verhalten zu verhelfen; es entsteht so zwecks einer Erziehung zur Nachhaltigkeit ein Mitspracherecht der Entscheider in ganz privaten Bereichen der Lebensführung der Mieter. Obwohl die Technologien, die den Sozialwohnungsträgern und den privaten Eigentümern angeboten werden, unterschiedlich sind, gehen sie doch alle von einer gemeinsamen Grundannahme aus, nämlich, dass es sich bei dieser Vorgehensweise um eine Win-win-Strategie handelt.

Hieraus kann ein erstes Postulat abgeleitet werden: dass es sich hier um eine Rezeption der Öko-Innovationen handelt, welche die Vielfalt der sozialen Kontexte ignoriert ${ }^{(35)}$ (insbesondere weil diese Rezeption gleichermaßen auch bereits eine praktische Nutzung bzw. Implementierung der Öko-Innovationen bildet). Wie aus dem Beispiel der in der Cité de l'Ill ausgeteilten Infobroschüren ersichtlich ist, setzen die Einbindungsmodalitäten der industriellen Technologien rationale Akteure voraus, was besonders beim Hinweis auf die erzielbaren Einsparungen bei entsprechender Verhaltensänderung bzw. Umstieg auf das neue Heizsystem deutlich wird. Dieses Social Engineering tut so, als ob die Mietergemeinschaft aus einer homogenen Gruppe bestehen würde, die in der Lage ist, all seine energetisch relevanten Verhaltensweisen zu steuern.

Zweitens tragen Dispositive wie die "Öko-Einstellung“, die einem Modell entsprechen, das von der ADEME ${ }^{(36)}$ landesweit propagiert wird, dazu bei, die Verantwortung auf die Bürger zu verlagern, anstatt die sozialen Unterschiede zu berücksichtigen. Diese Art der Dekontextualisierung steht im Widerspruch zu den Verbraucherstudien, die beweisen, dass es die finanziell besser gestellten Bevölkerungsschichten sind, die den größten Einfluss auf die Produktion von Treibhausgasen haben. Als Beispiel kann hier eine Studie des staatlichen französischen Statistik- und Wirtschaftsforschungsamtes genannt werden ${ }^{(37)}$ : In Frankreich benutzen über $70 \%$ der leitenden Angestellten und

34 Broschüre Rénov'Ill, Vermieter Habitation Moderne, 2013.

35 J.-B. Сомву, La question climatique (Anm. 2).

36 http://www.ademe.fr/particuliers-eco-citoyens/habitation/bien-gerer-habitat (letzter Zugriff am 19.11.2016).

37 INSEE, „Erhebung zur Ausstattung der Haushalte mit Haushaltsgeräten und Fahrzeugen unter Berücksichtigung der sozioprofessionellen Kategorien“, 2010, http://www.insee.fr/fr/themes/tableau. asp?ref_id=NATnon05135\&reg_id=0 (letzter Zugriff am 19.11.2016). 
oberen akademischen Berufe eine Spülmaschine, gegenüber 44\% der Arbeiter und 48\% der Angestellten. Auch bei der Mobilität sind die Unterschiede aufschlussreich: 52\% der leitenden Angestellten und oberen akademischen Berufe besitzen zwei Fahrzeuge, gegen 33\% der Arbeiter. In der zu beheizenden Wohnfläche offenbaren sich ebenfalls Unterschiede: Nicht-qualifizierte Arbeiter verfügen über weniger als $30 \mathrm{~m}^{2}$ pro Person, während die leitenden Angestellten und oberen akademischen Berufe $38 \mathrm{~m}^{2}$ beanspruchen. Die Anreizdispositive, welche auf der individuellen Verantwortung basieren, ignorieren den unterschiedlichen ökologischen Fußabdruck der verschiedenen sozialen Schichten in Bezug auf die Klimaerwärmung.

\section{Aneignungsungleichheiten bei den energetischen Herausforderungen und Technologien}

Wie auch in der Cité de l'Ill ist in Plobsheim der Einsatz von Anreizinstrumenten rund um Fachwissen und Technikkenntnisse organisiert. Die Diskrepanzen bei der Beteiligung der Bürger verweisen auf die Entstehung neuer sozialräumlicher Ungleichheiten.

Der Fragebogen beinhaltete 98 in fünf Themenbereiche aufgeteilte Fragen. Der erste Teil betraf die Beschreibung des Wohnraumes (Art des Wohnraumes, Fläche) sowie die Art des Energieverbrauchs (Heizungstyp usw.). Im zweiten Teil wurden die Verbrauchsgewohnheiten abgefragt, um die Problematik der Energiewende im Lichte der unterschiedlichen Lebensweisen zu betrachten: Sowohl die Ernährungsangewohnheiten (biologisch, Rolle von Qualitätslabels usw.) als auch die Mobilitätsgewohnheiten und der Bezug zur Natur (Besuch von Naherholungsgebieten, Freizeitaktivitäten im Freien) wurden erhoben. Im dritten Teil wurden die technischen Kenntnisse bezüglich der energetischen Innovationen und der verschiedenen Anlagen abgefragt (wie zum Beispiel der Unterschied zwischen Photovoltaik und Solarthermie), während weitere Fragen sich mit möglichen (bekannten oder unbekannten) Zukunftsszenarios befasst haben. Wir haben zum Beispiel die Befragten gebeten, uns ihre Einschätzung darüber zu geben, ob die erneuerbaren Energien in vierzig Jahren den landesweiten Energiebedarf decken könnten, oder wie realistisch sie ein Energieerzeugungsmodell einschätzen, dass vollständig auf Kernkraft verzichtet. Der vierte Teil variiert je nach Umfragegebiet. In der Cité de l'Ill ging es um die Umstellung des Heizungssystems und um das „Öko-Reflexe“-Sensibilisierungsprogramm. In Plobsheim haben wir die Motive der Einwohner untersucht, eine private Anlage zur Erzeugung regenerativer Energie zu installieren. Im letzten Teil haben wir uns mit dem sozioökonomischen Status der Befragten befasst, wie zum Beispiel dem Bildungsniveau, der sozioprofessionellen Kategorie, dem Familienstand usw.

Die zwei untersuchten Standorte unterscheiden sich in ihren sozioökonomischen Profilen ${ }^{(38)}$. Während in Plobsheim 2009 das durchschnittliche Netto-Einkommen eines steuerpflichtigen Haushalts bei $27.968 €$ lag, waren es in der Cité de l’Ill im selben Jahr lediglich $11.700 €$. Auch bei der Arbeitslosenquote gibt es große Unterschiede:

38 Cité de l'Ill ist ein eigener Census Tract, wie auch Plobsheim, so dass die sozioprofessionellen Kategorien stadteilgenau vorliegen: http://www.insee.fr/fr/methodes/zonages/iris/cartes/carte_iris_67482. pdf (letzter Zugriff am 19.11.2016). 
2010 waren es 4,3\% in Plobsheim gegen 16\% in der Cité de l'Ill. Ein weiterer Marker, die Verteilung der sozioprofessionellen Kategorien (Abbildung 1), weist auf einen höheren Anteil an Angestellten und Arbeitern in der Cité de l'Ill hin. Leitende Angestellte und obere akademische Berufen stellen dort weniger als 3\% der Einwohner, gegenüber 12\% in Plobsheim. Während in der Cité de l'Ill 100\% der Einwohner Mieter von Sozialwohnungen sind, sind es in Plobsheim lediglich 2\%. Zudem sind 80\% der befragten Plobsheimer Haus- oder Wohnungseigentümer. Die sozialen Unterschiede zwischen den beiden Gruppen werden auch bei den Bildungsabschlüssen sehr deutlich, insbesondere bei den höchsten und niedrigsten Abschlüssen (Abbildung 2).

Abbildung 1: Verteilung der Stichproben der Einwohner der Cité de l'Ill und von Plobsheim nach sozioprofessionellen Kategorien

(Neue Volkszählung des französischen Statistikamtes, INSEE, 2010) (gewichtete Stichprobe)

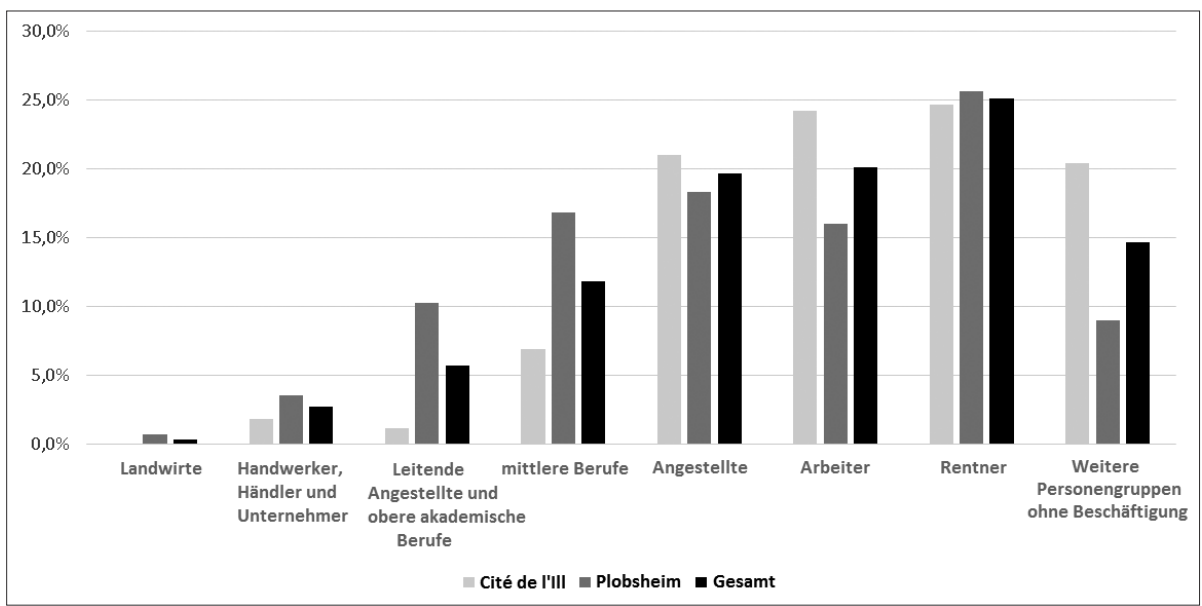

Abbildung 2: Verteilung nach Bildungsabschlüssen der Stichproben der Cité de l'Ill und von Plobsheim (gewichtete Stichprobe, N=300)

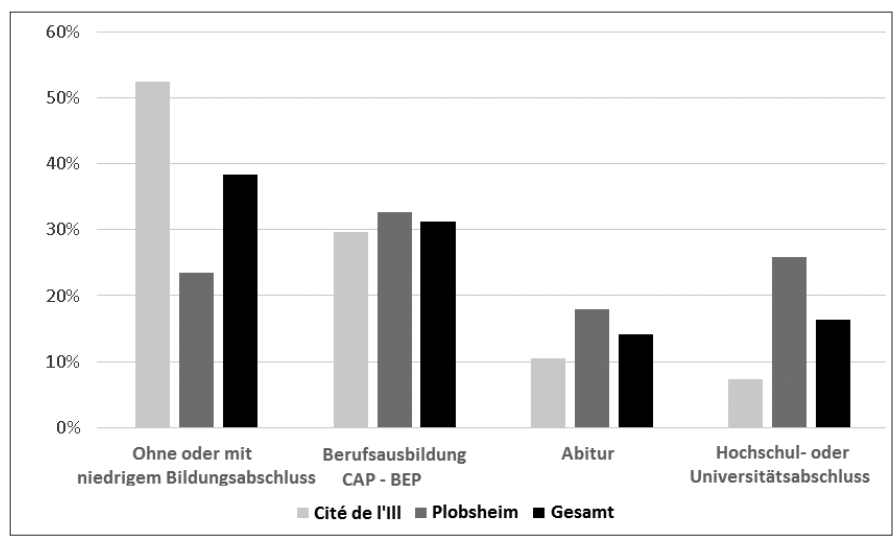




\section{Unterschiede bei der technischen Aneignung}

Der Fragebogen ermittelt die Kenntnisse, welche die Akteure von den verschiedenen Energiesystemen haben und betrachtet diese Wissensformen als Indikatoren für Aneignungsungleichheiten. Auf den ersten Blick scheinen die Unterschiede zwischen der Cité de l'Ill und Plobsheim nicht allzu groß: In Plobsheim erklären 74,2\% der Befragten, dass sie ausführliche Kenntnisse beim Thema Sonnenenergie haben und ebenfalls $74,2 \%$ geben an, ausführliche Kenntnisse beim Thema Windkraft zu haben, gegen 62,2\% und 53,2\% in der Cité de l'Ill (Abbildung 3). Die Sonnenenergie und die Windkraft haben den öffentlichen Raum erobert und werden in allen sozioökonomischen Schichten der Bevölkerung wahrgenommen. Genau betrachtet sind die Unterschiede zwischen Plobsheim und der Cité de l'Ill jedoch größer. Der Anteil der Einwohner, die nicht mit den technischen Begriffen vertraut sind (Geothermie, Biomasse, Solarthermie, Photovoltaik) - bzw. die im Fragebogen „keinerlei Vorstellung“ angekreuzt haben - ist in der Cité de l'Ill wesentlich größer, und zwar bei allen Formen erneuerbarer Energien (Abbildung 3). Je komplexer die Systeme zur

Abbildung 3: Wissensverteilung laut Angaben der Befragten zu den verschiedenen erneuerbaren Energien (gewichtete Stichprobe, N=300)

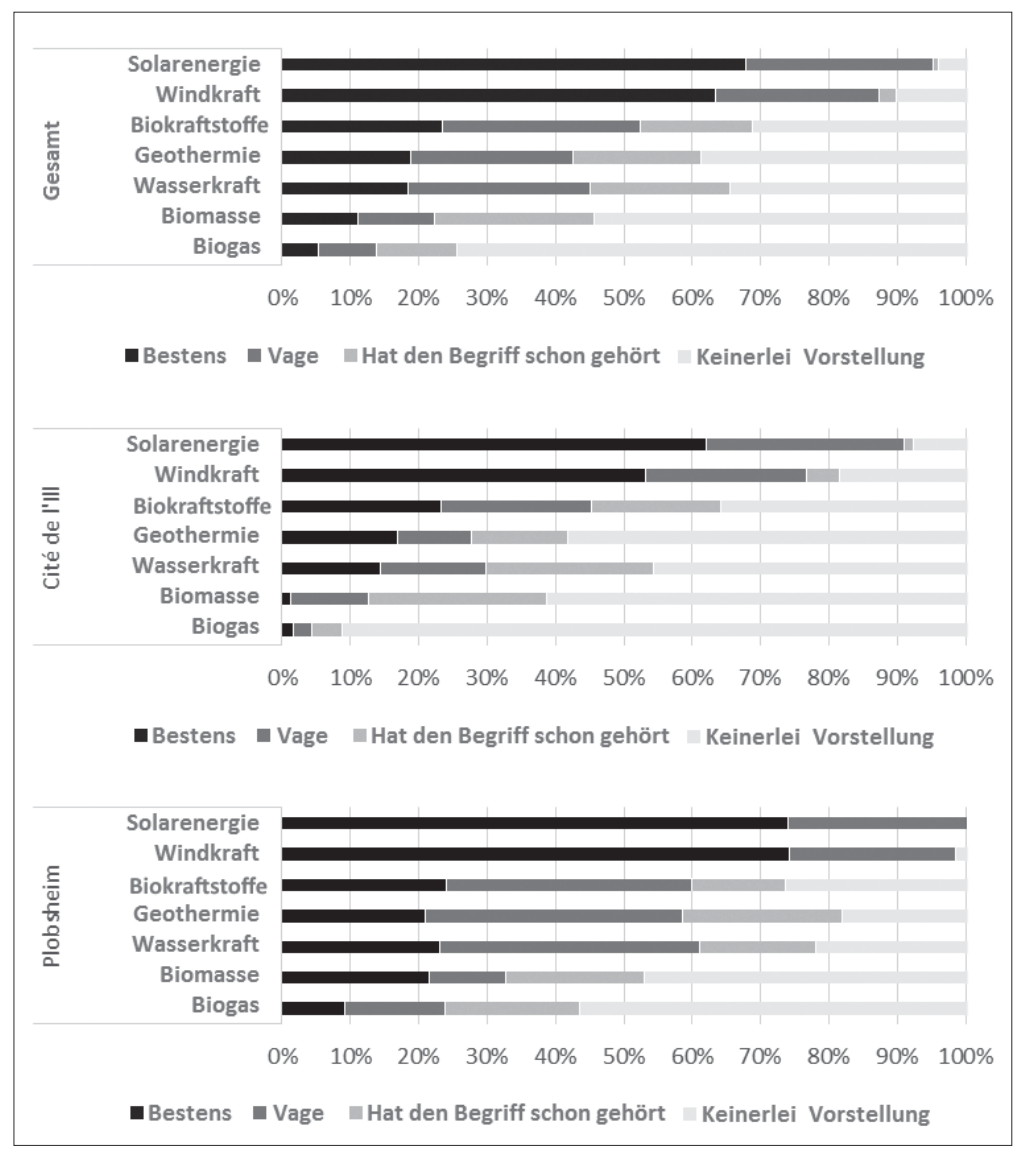


Energieerzeugung, desto größer der Unterschied zwischen den beiden Gruppen. Diese ungleichen Wissensstände lassen sich am Beispiel der Biogaserzeugung besonders gut ablesen: 90,7\% der Befragten der Cité de l'Ill geben an, „keinerlei Vorstellung“ davon $\mathrm{zu}$ haben, gegen 56,5\% in Plobsheim.

Die zur Realisierung der erneuerbaren Energiekonzepte verwendeten Technologien basieren auf einem technischen Modell, das den Bezugssystemen von Fachspezialisten entspringt. Dies erschwert die Beherrschung des Themengebiets. Um dies zu verifizieren, bezog sich eine Frage auf den Unterschied zwischen Wärmeerzeugung und Stromerzeugung aus Sonnenenergie. Sonnenenergie ist als Energiequelle zwar weitgehend bekannt, jedoch bleiben die Unterschiede zwischen Photovoltaik und Solarthermie für viele unklar. Die größten Wissenslücken bestehen in der Cité de l'Ill, sowohl bei denen, die behaupten, sich mit Sonnenenergie „bestens“ auszukennen, als auch bei denen, die angeben, nur „vage“ Bescheid zu wissen. Über 55\% der Befragten gaben an, „keinerlei Vorstellung“ über die Unterschiede zwischen den beiden Techniken zu haben, gegen circa 30\% der Plobsheimer Befragten (Abbildung 4).

Abbildung 4: Angaben zum Unterschied zwischen Solarthermie und Photovoltaik ( $\mathrm{N}=300)$

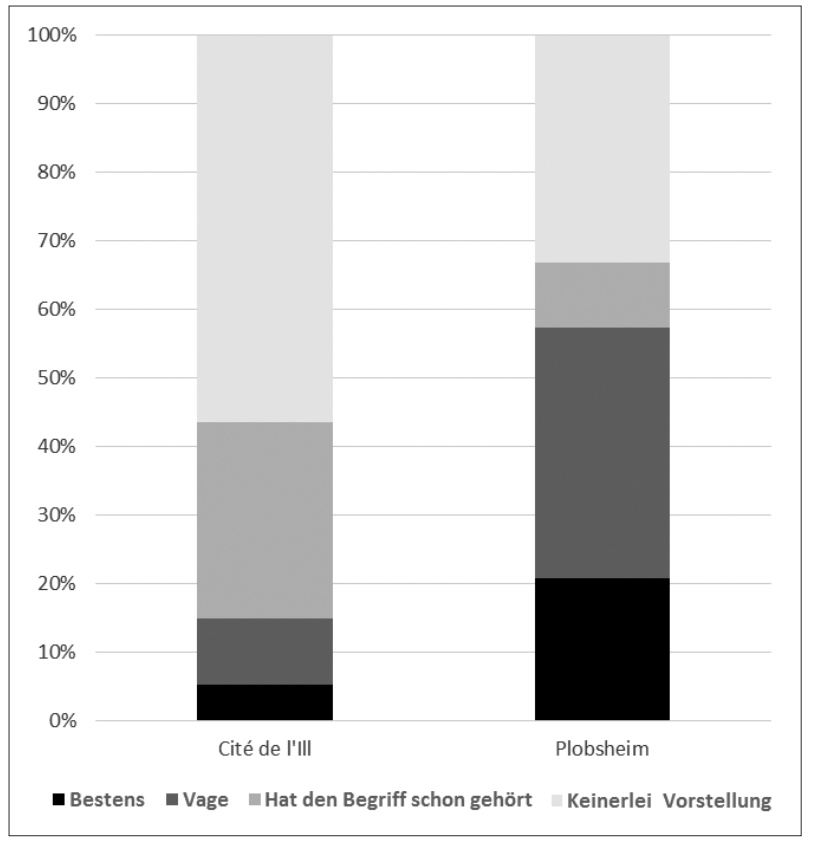

Eine weitere signifikante Variable sind die Energietypen, welche die Befragten mit erneuerbaren Energien in Verbindung bringen (Abbildung 5). Wir haben vier Produktionsmodi aufgezählt, und die Befragten gebeten, mit „ja“ oder „nein“ zu antworten. Dabei sind wir davon ausgegangen, dass diese Vorschläge zumindest einige der Befragten dazu bewegen, ihre Antworten nicht spontan zu geben, sondern die verschiedenen Items erst auf ihre Plausibilität zu überprüfen. Auch hier schneiden die meisten Sozialwohnungsmieter wieder schlechter als die Einwohner von Plobsheim ab 
(mit einer Ausnahme bei den Biokraftstoffen). So verbinden über 95\% der Plobsheimer Befragten die Stromerzeugung mit einer erneuerbaren Energiequelle (Sonnenenergie, Windkraft), gegen $60 \%$ in der Cité de l'Ill. Bestätigt wird dies durch eine weitere Antwort auf die Frage nach der aktuellen Relevanz der Kernenergie (unverzichtbar, wichtig, muss aufgegeben werden usw.): 27\% der Einwohner der Cité de l'Ill antworteten mit „weiß nicht“, gegen 1\% in Plobsheim.

Abbildung 5: Anteil der Befragten, der angegeben hat, dass dieser oder jener Energietyp durch eine erneuerbare Quelle produziert werden kann

(gewichtete Stichprobe, $\mathrm{N}=300$ )

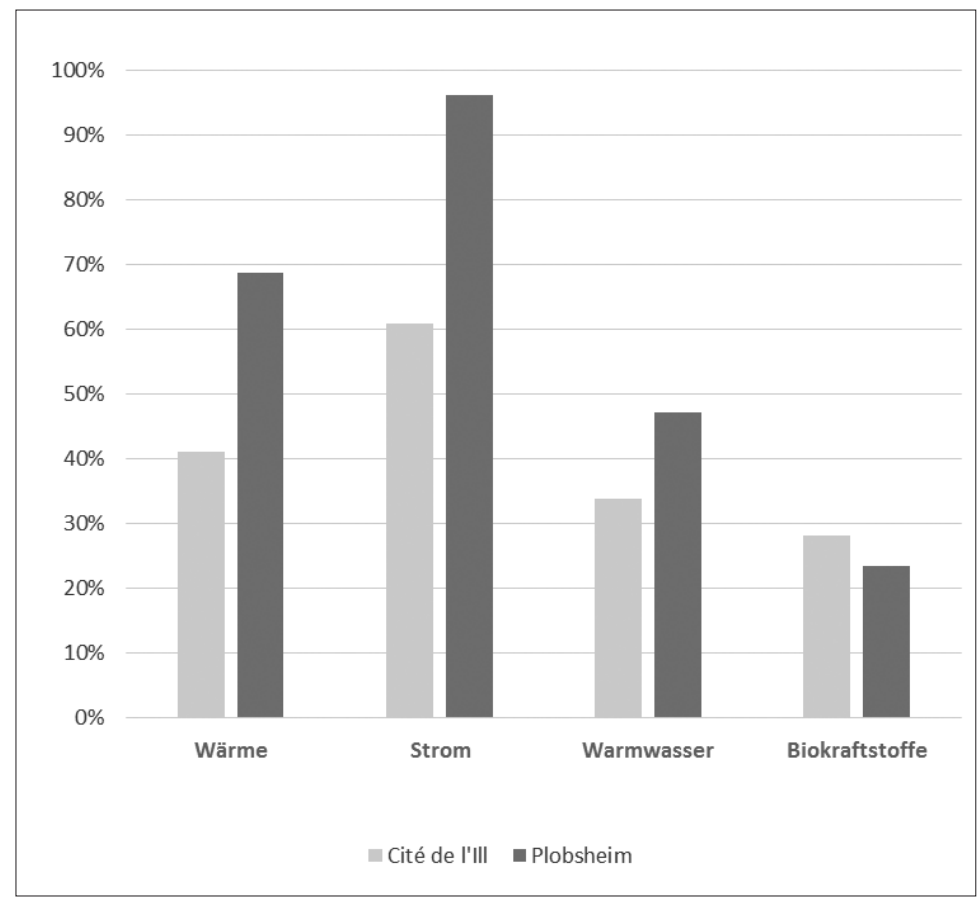

\section{Sozial bedingte Unterschiede bei der Akzeptanz}

In Verbindung mit den unterschiedlichen Aneignungsgraden beim technischen Wissen variiert auch die Akzeptanz der verschiedenen Technologien stark je nach sozialer Gruppe. Dabei offenbart sie eine unterschiedliche Beziehung zu den damit verbundenen Belastungen. Vor diesem Hintergrund haben wir den Befragten zwei Fotografien gezeigt, eine von einem Windpark und eine von einem Solarkraftwerk, und sie gefragt, ob sie den Bau einer solchen Anlage „sicher“, „wahrscheinlich“, „wahrscheinlich nicht“, oder „sicher nicht“ akzeptieren würden (Abbildung 6).

Es fällt auf, dass die Befragten in der Cité de l'Ill öfter bereit sind, eine Anlage in Sichtweite zu akzeptieren, als die Befragten in Plobsheim. Dies ist insbesondere der Fall bei dem Solarkraftwerk, das circa 10\% der Plobsheimer Befragten akzeptieren würden, gegen $30 \%$ in der Cité de l'Ill. Auch bei den Windrädern sind die Unterschiede deutlich: 32\% der Befragten in der Cité de l'Ill würden sie „sicher“ akzeptieren 
gegen weniger als 25\% in Plobsheim; und in der Cité de l'Ill zeigt die Umfrage ein überproportionales Auftreten von „weiß nicht“ Antworten (10\% gegen weniger als 5\% in Plobsheim). Die Tatsache, dass die sozial schwächeren Gruppen mit einer größeren Wahrscheinlichkeit eine solche Anlage in ihrer Sichtweite akzeptieren würden, korrespondiert mit den Ungleichheiten bei der Aneignung und den technischen Kenntnissen, die sich im Alltag in ökologischen Ungleichheiten manifestieren.

Abbildung 6: Akzeptanz von zwei Technologien zur Energieerzeugung durch die Einwohner von Plobsheim und der Cité de l'Ill

(gewichtete Stichprobe, $\mathrm{N}=300)(\mathrm{dept}=$ Departement)

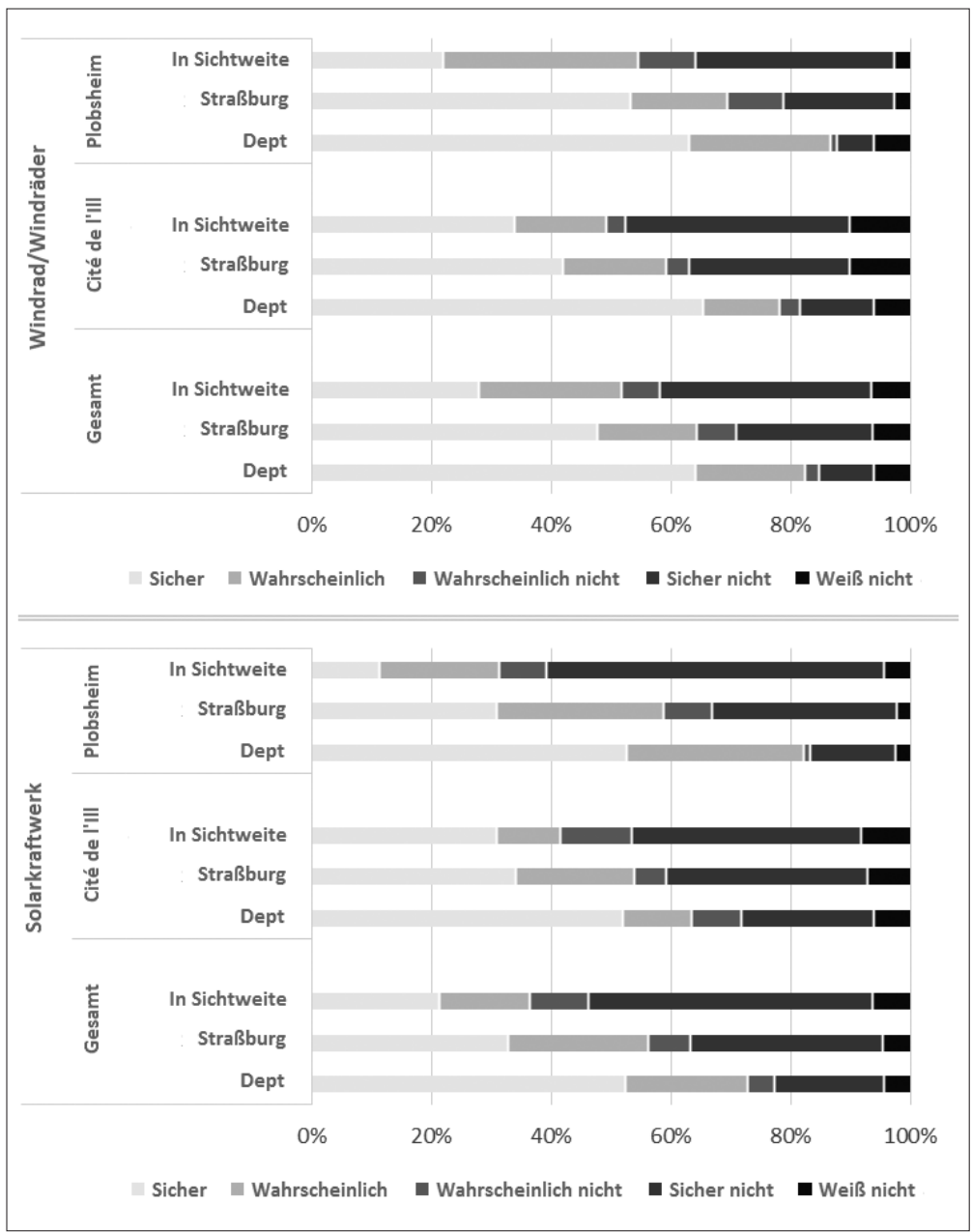

Geteilte Sorgen, doch ungleiche Möglichkeiten der Teilhabe

Die Veränderungen der Umwelt sind für beide befragte Gruppen Anlass zur Sorge: über 85\% der Befragten in der Cité de l'Ill und 88\% der Befragten in Plobsheim machen sich Sorgen über die Klimaerwärmung. Was die beiden sozialen Gruppen dabei 
unterscheidet, ist weniger der der Umwelt beigemessene Wert, sondern die Ungleichheiten bei den Mitgestaltungsmöglichkeiten, da diese die Fähigkeit voraussetzen, sich auf dem Gebiet der innovativen Energieerzeugungstechnologien auszukennen: „Das ist ja jetzt Mode, sich Sonnenmodule auf das Dach zu montieren, aber ich weiß nicht so recht, wozu das gut sein soll. Mir wurde gesagt, dass man die Sonnenenergie verkaufen oder nutzen kann“ (Interview, Einwohnerin der Cité de l'Ill, März 2014). Neben dem technischen Hemmnis stellt auch die schwer zu verstehende Reglementierung bei den Photovoltaikmodulen eine Hürde dar: „Das ist nicht leicht, mit den Solarmodulen, da muss man mit EDF reden, ich glaube, da muss man Anträge stellen, sie um Erlaubnis bitten, um seinen eigenen Strom produzieren zu dürfen“ (Interview, Einwohnerin der Cité de l’Ill, Mai 2013). Dieser Umstand kann man als „ökologische Ungleichheit" bezeichnen.

Zusammenfassend wird deutlich, dass das Engagement der Bürger nicht nur von einer positiven Einstellung gegenüber den erneuerbaren Energien abhängt, sondern vor allem von ihrem praktischen Wissen und ihren Kenntnissen des Energiesektors: Aus diesem Grunde ist ihre Fähigkeit zur Mitwirkung bei der Energiewende ungleich verteilt. Trotz einer insgesamt großen Befürwortung der erneuerbaren Energien zeigt die Statistik, dass die technischen Kenntnisse auf den beiden Standorten ungleich verteilt sind: so wissen nur 60\% der Befragten der Cité de l'Ill, dass man mit Solarenergie Strom erzeugen kann, gegen über 95\% in Plobsheim. Auch bei der Bereitschaft, sich zu engagieren, zeigen sich große Unterschiede, die sich bei Weitem nicht nur durch unterschiedliche Interessensschwerpunkte und Wertesysteme erklären lassen.

Allgemeiner gesehen, geht es darum die Gesamtheit der umweltbezogenen Verhaltensmuster und Lebensweisen sichtbar zu machen. Vergleicht man zum Beispiel die Ernährungsangewohnheiten anhand des Verzehrs von Bio-Lebensmitteln, stellt man erhebliche Unterschiede fest. In der Cité de l'Ill steht der Preis für $45 \%$ der Befragten an erster Stelle bei der Wahl der Lebensmittel und nur 1\% bevorzugen BioLebensmittel. Die Unterschiede zu Plobsheim sind signifikant: dort sind für 27\% der Einwohner Lebensmittel aus biologischem Anbau die erste Wahl. Weitere 14\% gaben „andere Qualitätslabels“ als ausschlaggebend beim Lebensmittelkauf an. Auch was den Zugang zur Natur die Freizeitaktivitäten im Freien angeht, sind die Unterschiede offensichtlich - sowohl bei der Häufigkeit der Ausflüge als auch bei den Ausflugszielen: $31 \%$ der befragten Plobsheimer nennen die Vogesen oder den Schwarzwald, gegen $2,6 \%$ der Befragten in der Cité de l'Ill. Letztere unternehmen eher kürzere Spaziergänge in der Nähe ihres Wohnsitzes - ein Hinweis auf Ungleichheiten bei dem Zugang und der Nutzung der „Natur“.

In diesem Sinne beleuchten wir die sozialen Ungleichheiten gegenüber der Instrumente, welche die Energiewende ermöglichen sollen, das Anreize schaffen sollen, sich dabei aber auf Wissensformen stützen, mit denen die Akteure in situ nicht unbedingt vertraut sind: Der Bezug zur Bürgerbeteiligung kann bestehende soziale Ungleichheiten verstärken oder kumulieren. Dabei haben sich in der Praxis Ungleichheiten bei der Partizipation oder bei der Motivation offenbart, oder unterschiedliche Fähigkeiten, die Relevanz von Umweltfragen zu begreifen.

Die Beziehung zwischen sozialen Ungleichheiten und Ökologie am Beispiel der Energiewende hat sich auf zweierlei Art manifestiert. Zunächst beziehen sich die 
eruierten Ungleichheiten nicht einfach auf die Wertevorstellungen der Einwohner, sondern auf die Beherrschung des nötigen technischen Instrumentariums der erneuerbaren Energien. Die Aneignung dieser Ermächtigungswerkzeuge setzt die Verfügbarkeit von Know-how und Kenntnissen voraus. Eine bloß partielle Kenntnis schränkt die Möglichkeiten der Einwohner ein, ihre Sorgen bezüglich der Umwelt, welche viele von ihnen teilen, in konkrete Handlungen umzusetzen. Folglich stellt die Verwendung spezialisierter Expertenbezugssysteme bei der Energiewende keinen urbanen oder sozialen Zusammenhalt her, wie er im Bezugssystem der nachhaltigen Entwicklung vorausgesetzt wird: Ganz im Gegenteil können die umweltbezogenen Ungleichheiten die ökonomischen und sozialräumlichen Ungleichheiten verstärken. Ein zweites Ergebnis betrifft die Art, mit der die erneuerbaren Energien eingeführt werden. In der Cité de l'Ill lässt das kollektive Regelwerk wenig Platz für individuelle Privatinitiativen. Die Bürgerpartizipation wird auf das Einsparen von Energie reduziert. Im Vergleich dazu wird in einem wohlhabenderen Rahmen wie Plobsheim die Energiewende mit weniger Zwangsmitteln eingeleitet. Der Aufruf zu mehr Sparsamkeit beim Energieverbrauch im Wohnraum wird bei sozial schwächeren Schichten mit mehr Nachdruck durchgesetzt (z. B. Maximaltemperatur von $20^{\circ} \mathrm{C}$ ).

\section{Diskussion}

Die Analyse trägt vor allem der environmental Justice im Allgemeinen und die Kritik des Partizipationsmodells im Besonderen bei, wodurch die Aufmerksamkeit auf die ökologischen und ökonomischen Ungleichheiten gelenkt wird. Das Fallbeispiel in zwei verschiedenen Wohngegenden in Straßburg zeigt, dass die gängige Praxis der Umsetzung der Energiewende diese Ungleichheiten verstärkt.

\section{Welche Einbindung der Einwohner in die Energiewende?}

Die Prozesse der Energiewende im urbanen Umfeld erscheinen vielfältig und wandelbar. In diesem Kontext stellt die „Wende“ keinen deutlichen Bruch mit dem Status quo dar, da die Reduzierung der $\mathrm{CO}_{2}$-Werte zu einem Bestandteil der Marktwirtschaft geworden ist. Wir haben gesehen, wie die Kontrolle dieser Themen von spezialisierten Akteuren übernommen wird, wie z. B. Energieversorger Idex. Die Umstellung auf erneuerbare Energien liegt hauptsächlich bei existierenden Industrien und Dienstleistern, und die Suche nach Mittelwegen zwischen Sozialwohnungsträgern, öffentlicher Hand und Unternehmen aus dem Energiesektor zwecks Bedienung aller Interessen kann letzten Endes zu einer Ausgrenzung der Einwohner führen, die nicht zu diesen Spezialistengruppen gehören. Die marktbeherrschenden Akteure bewahren ihre Stellung, indem sie das Konzept der nachhaltigen Energieerzeugung durch erneuerbare Quellen in ihren Diskurs aufnehmen; dies geht einher mit politischen Entscheidungen des Energiewandels, welche sich stärker auf das Angebot als auf die Nachfrage fokussieren, und dies durch eine entsprechende Anreizpolitik unterstützen; dabei wird über die Dimension der Verbrauchs- und Lebensgewohnheiten trotz ihrer großen sozialen Bedeutung hinweggesehen.

Die Teilhabemöglichkeiten der Bürger sind umso beschränkter, als die Governancemodelle der städtischen Projekte zur Einführung erneuerbarer Energien zwischen dezentralisierten Konzepten, Zwangslogiken und einer Professionalisierung und 
Normierung der Partizipationsmodalitäten schwanken ${ }^{(39)}$. Der Vergleich zwischen Plobsheim und der Cité de l'Ill verrät ungleiche Mitwirkungsgrade: Aus dem Blickwinkel des Einzelnen wird die Einführung der erneuerbaren Energien vor allem durch technische Innovationen ermöglicht, welche die sozioökonomischen Unterschiede weiter verstärken, vor allem den Unterschied zwischen Eigentümern und Mietern. Während die Installation von PV-Anlagen im Falle von Eigentümern weitgehend ohne Abstimmung mit den Nachbarn erfolgen kann, handelt es sich bei einem Nahwärmenetz um eine Technologie, die mit einem sehr viel größeren Abstimmungsaufwand verbunden ist. Damit zeichnet sich ab, dass unterschiedliche Partizipationsgrade technisch wie auch sozial bedingt sind. Zugleich ist es ein Zeichen der Prozeduralisierung der Einwohnerpartizipation, die im Rahmen des Nachhaltigkeitsdiskurses vorangetrieben wird: Die Energiepolitiken enthalten Ad-hoc-Dispositive, deren Rolle es ist, die Verbraucher zu sozialisieren. Die „Öko-Reflexe“, deren Ziel die Verbrauchsreduzierung ist, oder die Abnahmegarantie mit Mindestpreis für den von Privatpersonen erzeugten Ökostrom, verstärken noch diesen Normierungseffekt. So beruhen zum Beispiel die Infobroschüren, die an die Sozialwohnungsmieter in der Cité de l'Ill vergeben werden, auf leicht zu übernehmende Bezugssysteme der Energieverbrauchsreduzierung. Die Partizipation der Einwohner ist auf die Annahme des Instrumentes beschränkt, obwohl es die Vielfalt der sozialen und urbanen Räume, in denen die Innovationen stattfinden, ignoriert.

In der Cité de l'Ill beschränken die Anreizinstrumente die Partizipation der Einwohner auf eine Reduzierung ihres Energieverbrauchs. Offen bleibt das Nachdenken über eine andere Dynamik der Wende, die sich auf ein Register gemeinsamen Wissens basieren würde, z.B. die Notwendigkeit, ökologische Initiativen zu entwickeln, welche sich die Einwohner leicht aneignen können - was voraussetzt, dass diese neuen Lösungsansätze sie auch miteinbeziehen. Oft wird also implizit die Behauptung aufgestellt, dass es sich bei Partizipation bzw. Partizipationschancen um ein gesellschaftlich wertvolles Gut handle, das von allen gleichermaßen erwünscht sei. In der Tat sind die befragten Haushalte nicht in gleicher Weise über alternative Energien informiert, und engagieren oder interessieren sich auch nicht in gleicher Weise für PV-Anlagen bzw. Wärmenetze. Der Partizipationsrahmen ist eigentlich durch die Behörden finanziell oder institutionell vorgegeben. Da wirken sich Fähigkeiten und Möglichkeiten auf die Ungleichheit aus - und so erscheint das Verhältnis zwischen sozialer und ökologischer Ungleichheit.

\section{Auf dem Weg zu einer Neudefinition der Energiewende?}

Im Lichte des ständigen Spannungsfeldes zwischen technischen und ökonomischen Herangehensweisen auf der einen Seite und ökologischen und sozialen auf der anderen $^{(40)}$, offenbaren unsere Analysen die Notwendigkeit einer Resubstantialisierung der sozialen Bindung und eines kollektiven Projektes des Wandels ${ }^{(41)}$. Infrage

39 Christen/Hamman/Jehling/Wintz, Erneuerbare Energien-Systeme (Anm. 11); Christen/HamMAN, Transition énergétique et inégalités environnementales (Anm. 17).

40 Hamman/Anquetin/Monicolle, „Contemporary Meanings of the ,Sustainable City““ (Anm. 7).

41 Ian BAiley, Geoff A. Wilson, „Theorising Transitional Pathways in Response to Climate Change: Technocentrism, Ecocentrism, and the Carbon Economy“, Environment and Planning A, 41/10 (2009), S. 2324-2341; Choné/Hajek/Hamman, Guide des Humanités environnementales (Anm. 22). 
steht die Rolle der Politik beim Thema Nachhaltigkeit ${ }^{(42)}$. Wir sind mit einer ganzen Grammatik der Legitimation konfrontiert, welche die Grundlage aller Diskurse zur Nachhaltigkeit bildet ${ }^{(43)}$. Wenn von einem „einfachen“, „erweiterbaren“, „intelligenten“ usw. Dispositiv die Rede ist, ist damit die Weiterentwicklung in Richtung einer managementgesteuerten Vorgehensweise in einer technisch geprägten und kollektiven "Risikokultur“ gemeint ${ }^{(44)}$, in der es darum geht, sich zu schützen (indem man „besser integriert“ oder „anpassungsfähiger“ usw. ist). Dieses instrumentelle Bezugssystem der Nachhaltigkeit hat sich als dominantes Modell etabliert, mit begleitenden Maßnahmen, die zu "positiven“ Verhaltensweisen ermahnen, vor allem was den Energieverbrauch angeht. Das haben das kollektive Heizungssystem der Cité de l'Ill und die privaten Erzeuger "grüner Energien“ in Plobsheim gezeigt. Man befindet sich nicht einfach nur in einem technischen Repertoire oder in einer Aufforderung zu Handeln; es handelt sich um einen ganzen Diskurs über das Soziale und des Sozialen, aus dem die Verbindung zwischen Energiewende und ökologischen Ungleichheiten hervortritt. Sich für die erneuerbaren Energien zu interessieren, ist für den Durchschnittsbürger keine Selbstverständlichkeit. Hierbei geht es nicht nur um Ungleichheiten bei dem der Umwelt und der Nachhaltigkeit entgegengebrachten Interesse ${ }^{(45)}$; die Einwohner sind auch mit Ungleichheiten bei den lokalen Mitwirkungsmöglichkeiten konfrontiert, im Sinne von Amartya Sen ${ }^{(46)}$. Dies kann zu verstärkten Ungleichheiten führen, indem die Akteure daran gehindert werden, an der Erschaffung eines Gemeinguts teilzunehmen - obwohl dies ein zentrales Element einer geteilten Politik der ökologischen Wende darstellt ${ }^{(47)}$.

\section{Zusammenfassung}

Dieser Artikel befasst sich mit den umweltbezogenen sozialen Ungleichheiten, welche aus den unterschiedlichen Fähigkeiten der einzelnen Bürger entstehen, sich im Rahmen der Energiewende beim Thema erneuerbare Energien einzubringen. Der Artikel fußt auf qualitativen und quantitativen Feldstudien im urbanen Umfeld, die zwischen 2012 und 2014 im Stadtgebiet Straßburg (Frankreich) durchgeführt wurden. Wir zeigen, dass

42 E. Swyngedouw, „The Antinomies of the Postpolitical Cities“ (Anm. 1).

43 Ortwin Renn, Jürgen Deuschle, Alexander Jäger, Wolfgang Weimer-Jehle, Leitbild Nachhaltigkeit: Eine normativ-funktionale Konzeption und ihre Umsetzung, Wiesbaden, VS Verlag für Sozialwissenschaften, 2007; Gabriele Dürвеск, Urte Sтовве (Hg.), Ecocriticism. Eine Einführung, Köln, Böhlau, 2015; Aurélie Choné, Isabelle Hajeк, Philippe Hamman (Hg.), Rethinking Nature, Challenging Disciplinary Boundaries, New York/London, Routledge, 2017.

44 Anthony Giddens, Modernity and Self-Identity, Stanford, Stanford University Press, 1991.

45 HAMmAN/FrAnk/MANGOLD, „Les trajectoires de conversion écologique face aux enjeux socio-économiques“ (Anm. 33).

46 Amartya Sen, Inequality Reexamined, Oxford, Clarendon Press, 1992; Emily Schultz, Marius Christen, Lieske Voget, Paul Burger, „A Sustainability-Fitting Interpretation of the Capabilities Approach: Integrating the Natural Dimension by Employing Feedback Loops“, Journal of Human Development and Capabilities, 14 (2013), H. 1, S. 115-133.

47 Joan Martinez-Alier, The Environmentalism of the Poor: A Study of Ecological Conflicts and Valuation, Cheltenham, Edward Elgar, 2002. 
sich hinter dem im Vordergrund stehenden Diskurs der „Bürgerpartizipation“ in Wirklichkeit „Ökoinnovationen"verbergen, die aus Zwängen und Vorschriften bestehen, und dass die unterschiedlichen Beziehungen der Einwohner zu den technischen Instrumenten, welche die Einführung erneuerbarer Energien ermöglichen sollen, die Grundlage von ökologischen Ungleichheiten bilden, welche die sozio-ökonomischen Gräben in der Stadt weiter vertiefen.

\section{Résumé}

Cet article interroge les inégalités sociales face à l'environnement, à travers les capacités des citoyens à se mobiliser autour des dispositifs d'énergies renouvelables dans le cadre de la transition énergétique. Il s'appuie sur des enquêtes de terrain, qualitatives et quantitatives, à l'échelle urbaine, conduites de 2012 à 2014 au sein de l'aire métropolitaine de Strasbourg (France). Nous montrons que le registre de l'implication habitante, mis en avant, masque des "éco-innovations" relevant en fait de prescriptions; et qu'à partir des rapports différenciés des habitants face aux instruments techniques censés matérialiser les énergies renouvelables, des inégalités écologiques renforcent les lignes de partage socio-économiques dans la ville.

\section{Summary}

This paper deals with socio-environmental inequalities regarding the capacity of citizens to mobilize action on renewable energy as part of the energy transition. It is based on field surveys (qualitative and quantitative) on an urban scale, conducted from 2012 to 2014 in the Strasbourg metropolitan area (France). We show that the often highlighted register of resident participation actually masks prescriptive regulatory "eco-innovations"; and that the differentiated perceptions of the inhabitants facing the technical tools supposed to materialize renewable energy explain environmental inequalities that reinforce socio-economic dividing lines in the city. 